\title{
Arthroscopic confirmation of femoral button deployment prevents soft tissue interposition in $\mathrm{ACL}$ reconstruction
}

\author{
Sylvain Guy ${ }^{1}$ - Alessandro Carrozzo ${ }^{1} \cdot$ Alexandre Ferreira $^{1} \cdot$ Thais Dutra Vieira $^{1}$ (1) - Benjamin Freychet ${ }^{1}$. \\ Mathieu Thaunat ${ }^{1} \cdot$ Bertrand Sonnery-Cottet $^{1}$
}

Received: 17 May 2021 / Accepted: 27 September 2021 / Published online: 19 October 2021

(c) European Society of Sports Traumatology, Knee Surgery, Arthroscopy (ESSKA) 2021

\begin{abstract}
Purpose The purpose of this study was to determine whether direct arthroscopic control of femoral buttons can prevent improper deployment and soft tissue interposition in anterior cruciate ligament (ACL) reconstruction.

Methods A retrospective analysis of prospectively collected data from the SANTI study group database was performed. All patients who underwent ACL reconstruction using suspensive femoral fixation between 01/01/2017 and 31/12/2019 were included. Patient assessment included demographics, sports metrics, reoperations performed and femoral button-related specific complications such as iliotibial band (ITB) irritation and/or septic arthritis. Proper deployment of the button and soft tissue interposition were assessed on postoperative radiographs.

Results A total of 307 patients underwent ACL reconstruction using adjustable femoral button fixation and were analyzed after a mean follow-up of $35.2 \pm 11.0$ months (14.3-50.2). The mean age was 39.5 \pm 10.9 -years old (range 13.3-70.6). Postoperative radiographs showed a correctly deployed femoral button without soft tissue interposition for all patients. No septic arthritis was reported. Nine patients (2.9\%) suffered from lateral pain related to ITB irritation due to the button. Five of them had their symptoms resolve during rehabilitation. Ultrasound-guided corticosteroid infiltration was necessary for four patients after an average delay of $14.5 \pm 4.8$ months (11.7-21.7). Three patients were then symptom-free, but one required surgical removal of the implant 27.5 months after the surgery. Regarding unrelated femoral button complications, 15 patients (4.9\%) underwent secondary arthroscopic procedures, including meniscectomy (1.6\%), surgery for cyclops syndrome (2.6\%) and revision ACLR $(0.7 \%)$.
\end{abstract}

Conclusion Arthroscopic confirmation of femoral button deployment prevents soft tissue interposition without specific complications.

Level of evidence Level IV.

Keywords ACL $\cdot$ ACL reconstruction $\cdot$ Femoral button $\cdot$ Button deployment $\cdot$ Tissue interposition $\cdot$ Ilio-tibial Band Irritation

\section{Introduction}

Graft fixation during anterior cruciate ligament reconstruction (ACLR) is a hotly debated topic [1,2]. Since the study by Kurosaka et al.[3] in 1987, several hundred studies based mainly on in vitro biomechanical experiments have been devoted to this subject [1]. Given the lack of clinical studies

Thais Dutra Vieira

thaisdutravieira@hotmail.com

1 Ramsay Générale de Santé, Hôpital Privé Jean Mermoz, Centre Orthopédique Santy, 24 Avenue Paul Santy, 69008 Lyon, France demonstrating the superiority of one method over another, no gold standard has been recommended to date. Easy and fast to use while providing excellent clinical outcomes, suspensive femoral fixation has become a popular technique over the last fifteen years [4-7].

Anterior cruciate ligament (ACL) graft fixation using a femoral button presents specific complications, such as improper deployment of the implant $[8,9]$, widening of the tunnel [10-12], and iliotibial band (ITB) irritation [13, 14]. Occurring in $15-25.2 \%$ of cases, soft tissue interposition is, however, the most frequent complication [9, 15-17]. A degradation of postoperative clinical scores is also directly related to implant migration over time [15, 18]. As suggested 
by Mae et al.[15], this could decrease femoral fixation quality and alter graft integration. The increased rate of graft rupture in cases of ACL reconstruction with a femoral button compared to other fixation modes, as reported by the Danish and Norwegian registries, could then be explained $[4,6]$.

Various techniques have been described to prevent these complications, such as intraoperative fluoroscopy or accessory approaches allowing direct visualization of the implant $[9,19]$. Although effective, these methods are time consuming and either irradiating or invasive. Intra-articular positioning of the femoral tunnel exit point, made possible with outside-in drilling, allows direct arthroscopic visualization of the femoral button in the lateral glutter [20,21]. The purpose of this study was to determine whether soft tissue interposition and improper button deployment can be prevented using direct arthroscopic control. The hypothesis was that direct arthroscopic visualization of femoral button deployment decreases specific complications related to femoral fixation during ACLR.

\section{Materials and methods}

Institutional Review Board approval (COSRGDS-2021-01-004) was granted for this study, and all participants gave valid consent to participate. A retrospective analysis of prospectively collected data from the SANTI study group database was performed. All patients who underwent arthroscopic ACLR (primary or revision) or ACL repairs performed by a single surgeon (BSC) from 01/01/2017 to $31 / 12 / 2019$ were assessed for eligibility. The diagnosis of ACL tears was based on magnetic resonance imaging (MRI) and a complete clinical examination. Surgical management was decided upon when the patient's subjective instability restricted him in his sports or daily activities. From this group, all patients whose ACL graft was fixed to the femur using suspensive fixation (TightRope ${ }^{\circledR}$, Arthrex, Naples, Florida) were identified and included. Patients who underwent ACL repairs or major concomitant surgery (e.g., multiligament reconstructions) were excluded.

\section{Data extraction}

Demographic data (age, sex, weight, height and body mass index (BMI)) and the characteristics of the index surgical procedures (whether a LEAP or associated high tibial osteotomy was performed or meniscal and chondral lesions were observed during arthroscopy) were extracted from the SANTI study group database. Preoperative sports practice was reported, and sports levels were evaluated by the Tegner activity scale.

Knee radiographs were systematically performed postoperatively. Each radiograph was analyzed by a trained orthopedic surgeon who attested to the correct deployment of the button on the lateral side of the femur. The distance between the cortex and the implant was measured on frontal radiographs. If the latter distance was less than one millimeter, soft tissue interposition was considered nonexistent [15].

Data regarding subsequent arthroscopy and its indications were provided. Any postoperative complications that did not require surgical management were also indicated, as was the resulting treatment. Potentially femoral button-related complications were given particular attention: lateral knee pain (requiring corticosteroid infiltration or not), surgical removal of the implant, and septic arthritis.

\section{Surgical technique}

All ACLRs were performed according to the Single-AnteroMedial Bundle Biological Augmentation (SAMBBA) technique described previously [20]. The semitendinosus (ST) was harvested using an open-ended tendon stripper and then quadrupled. The tibial insertion was preserved to improve fixation and vascularity [22]. An outside-in femoral guide was used, and its exit point was positioned intraarticularly under arthroscopic control. A 20-mm femoral bone socket was created using a FlipCutter II (Arthrex, Naples, Florida) according to the graft diameter [23]. Soft tissues were shaved along the lateral end of the femoral tunnel. The ACL graft was passed from the tibia to the femur. The arthroscope was introduced into the lateral gutter, allowing direct visualization of the button deployment (Fig. 1). The graft was secured on the femoral

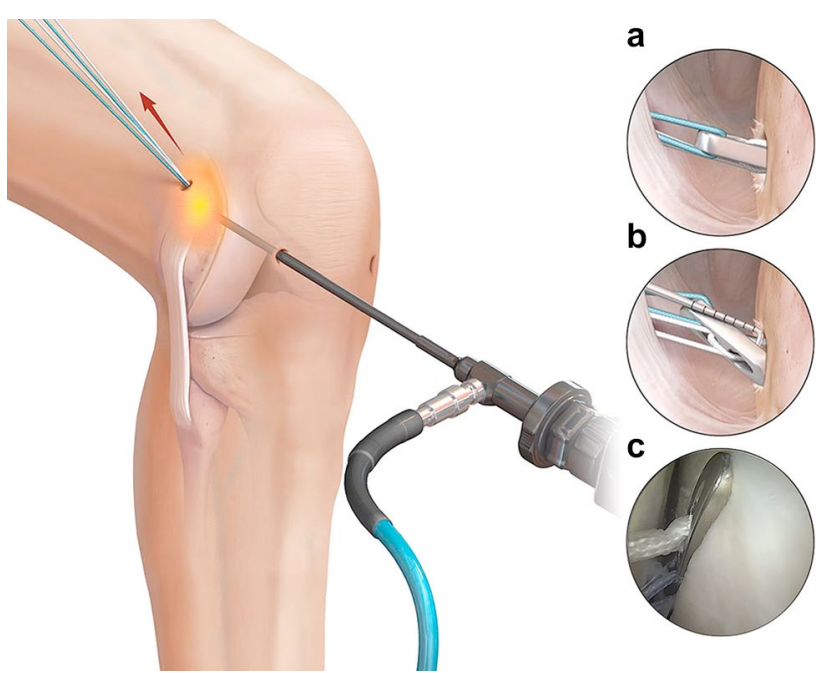

Fig. 1 Arthroscopic confirmation of correct Button deployment in the lateral gutter. Process of flipping the TightRope button on the lateral cortex of the femur under arthroscopic visualization in the right knee. While the arthroscope is in the lateral gutter, the blue suture is tensioned to pull the graft into the joint. a The button is visualized while exiting the tunnel. b, $\mathbf{c}$ A probe is used to help seat the button. (Reprinted with permission from Arthroscopy Techniques) 
side with the TightRope ${ }^{\circledR}$ tensioning device (Arthrex, Naples, Florida) and then fixed on the tibial side at $30^{\circ}$ of flexion using a bioabsorbable screw (Arthrex, Naples, Florida). The button sutures were cut flush with the implant under arthroscopic control without additional safety knots.

For patients who underwent an additional lateral extra-articular procedure (LEAP), a modified deep Lemaire procedure [24] or independent reconstruction of the anterolateral ligament using the gracilis tendon was performed.

\section{Rehabilitation}

Weight bearing was allowed in the immediate postoperative period and while protected by crutches until the fourth week. Mobilization of the knee was brace free, except in the presence of meniscal repairs; weight bearing was progressive. Early rehabilitation focused on the restoration of full extension. Open-chain muscle strengthening was contraindicated until 3 months postoperatively. Cycling was allowed at one month, running at three months, nonpivoting sports at 4 months, pivoting noncontact sports at 6 months and pivoting contact sports at 8 to 9 months.

\section{Follow-up}

Postoperative follow-up was undertaken either by a sport physician or the senior surgeon at 3 and 6 weeks and then at 3, 6, 12 and 24 months. Anteroposterior side-to-side laxity (Rolimeter ${ }^{\circledR}$, Aircast, Europe) and pivot shift examinations were performed at each consultation, and the occurrence of femoral button-related lateral pain was assessed. If the patient's pain did not improve through functional rehabilitation, echo-guided corticosteroid infiltration was performed around the implant by a trained radiologist. In case of failure, surgical removal of the button was then proposed.

\section{Statistical analysis}

Descriptive statistics were calculated to characterize demographics, surgical characteristics, and radiographs analysis, including proper button deployment and soft tissue interposition. Additional descriptive statistics were obtained regarding postoperative complications, stratified by their potential connection to the femoral button. Quantitative variables are expressed as the mean values, standard deviation, and range. For qualitative variables, the number of observations and the percentage are presented.

\section{Results}

A total of 1773 ACLRs and ACL repairs were performed by the same senior surgeon between 01/01/2017 and 12/31/2019. Among these, 374 grafts were fixed to the femur using suspensive fixation (TightRope ${ }^{\circledR}$, Arthrex, Naples, Florida). One patient was excluded because of concomitant multiligament reconstruction. Sixty-six ACL repairs were also excluded. Therefore, the results of 307 ACLRs performed according to the SAMBBA technique on 302 patients were retained for analysis. The study flow chart is shown in Fig. 2.

The demographics of the study population are presented in Table 1. The mean age was 39.5 \pm 10.9 -years old (13.3-70.6), with a mean follow-up of 35.2 111.0 months (14.3-50.2). A majority of patients were involved in pivoting or pivotingcontact sports ( $n=239,77.9 \%$ ) before surgery, with a mean Tegner activity scale score of $6.0 \pm 1.2(3-9)$. The surgical characteristics are presented in Table 2 . Twenty-six patients (8.5\%) had a concomitant lateral extra-articular procedure (LEAP), either modified Lemaire $(n=14,4.6 \%)$ or anterolateral ligament reconstruction (ALLR, $n=12,3.9 \%$ ). Meniscal lesions were identified and treated in 143 patients $(46.5 \%)$, most of which involved the medial meniscus $(n=103,33.5 \%)$.

The mean side-to-side laxity (Rolimeter ${ }^{\circledR}$, Aircast, Europe) was $0.51 \pm 0.89 \mathrm{~mm}$ ( -4 to 3 ). Postoperative radiographs showed a correctly deployed femoral button without soft tissue interposition in all patients (Fig. 3). Regarding potentially button-related complications, nine patients $(2.9 \%)$ suffered from lateral pain related to ITB irritation (Table 3). Five of them had their symptoms resolve during rehabilitation. Ultrasound-guided corticosteroid infiltration was necessary for four patients after an average delay of 14.5 months. Three patients were then symptom free. Nevertheless, one 45-year-old female patient, a sports education teacher, had persistent pain. She underwent ACLR in January 2019 following a work-related accident and experienced ITB irritation during follow-up. Corticosteroid infiltration was performed in January 2020. Due to ineffective medical treatment, surgical removal of the femoral implant was scheduled in March 2020 but could not be performed until May 2021, 27.5 months after surgery, due to the coronavirus disease 2019 (COVID-19) pandemic. Regarding other complications, 15 patients $(4.9 \%)$ underwent secondary arthroscopic procedures, including meniscectomy (1.6\%), surgery for cyclops syndrome $(2.6 \%)$ and revision ACLR $(0.7 \%)$ (Table 4). 
Fig. 2 Study flow chart. Study flow chart in line with the Strengthening the Reporting of Observational Studies in Epidemiology (STROBE) statement [40]. ${ }^{\mathrm{a}} A C L$ anterior cruciate ligament, $A C L R$ anterior cruciate ligament reconstruction
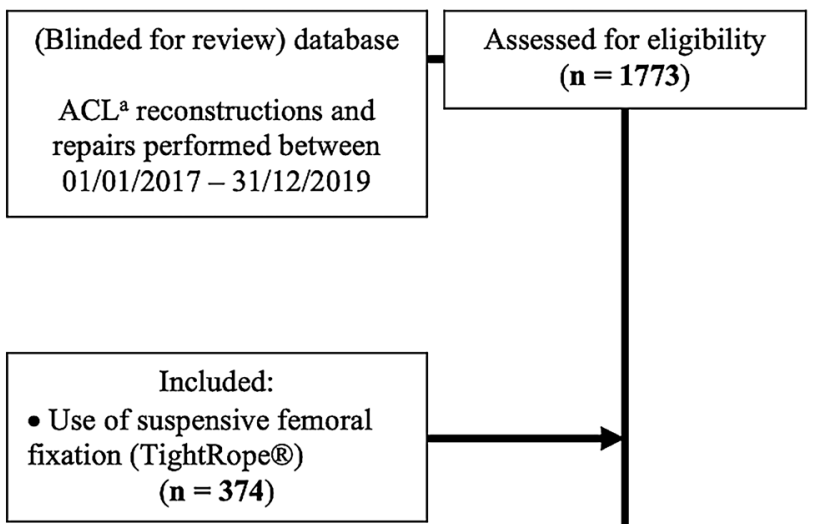

\section{Discussion}

The main finding of this study was that intra-articular positioning of the femoral button in ACLR, allowing direct arthroscopic visualization of the implant, prevented improper deployment and soft tissue interposition. Preventing these complications was not achieved at the expense of other complications. No septic arthritis was reported in our cohort. Only 9 patients (2.9\%) experienced lateral knee pain related to ITB irritation, and one $(0.3 \%)$ required implant removal. Our initial hypothesis, is therefore, validated.

Suspensory femoral fixation has become increasingly attractive to surgeons over the last fifteen years [4-7, 25]. Pitfalls have, however, been reported, one of the most criticized being postoperative widening of the tunnels [10-12, $26,27]$. No study has yet demonstrated an unfavorable clinical impact on either subjective scores or objective stability $[10,11,26,27]$. The hypothesis that this phenomenon is related to the distance between the graft and its fixation was questioned by Choi et al. as no correlation was found between the adjustable femoral button loop length and tunnel widening [28]. Ma et al. even reported greater widening when bioabsorbable interference screws were used [29]. Finally, Pioger et al. showed no influence of tunnel widening on the surgical management of revision ACLR [30].
Single-stage management has always been possible using an outside-in femoral guide, and excellent clinical outcomes have thus been obtained independently of preoperative tunnel position and width. This potential complication of the femoral button should not then call its use into question.

Other complications have been reported, such as improper deployment of the button in the femoral tunnel or its intraarticular migration over time $[8,31,32]$. These complications were mentioned in case reports and had no clinical consequences. More detrimental from a functional point of view, ITB irritation related to the implant has been reported $[13,14]$. Both Taketomi et al. and Kawaguchi et al. performed surgical removal of femoral buttons in all patients with disabling lateral pain. [13, 14] Implant positioning too close to the lateral epicondyle or soft tissue interposition were identified as potential risk factors. In the present study, the use of an outside-in femoral guide allowed us to set the button distant from the epicondyle, and systematic shaving of soft tissues along the lateral end of the femoral tunnel limited the interposition of soft tissue beneath the implant. Moreover, no additional safety knot was performed on the button, thus limiting its volume beneath the ITB. We nevertheless, observed nine patients with ITB irritation (2.9\%), but only one required removal of the material $(0.3 \%)$. 
Table 1 Demographics of the study population and sport practice metrics

\begin{tabular}{ll}
\hline & SAMBBA $n=307$ \\
\hline Sex $n(\%)$ & \\
Male & $181(59.0)$ \\
Female & $126(41.0)$ \\
Age & $39.5 \pm 10.9(13.3-70.6)$ \\
mean \pm SD (range), y & \\
BMI $^{\mathrm{a}}$ & $24.5 \pm 3.6(16.6-36.8)$ \\
mean \pm SD (range), kg/m ${ }^{2}$ & \\
Side $n(\%)$ & $153(49.8)$ \\
Left & $154(50.2)$ \\
Right & $12.5 \pm 38.1(0.2-329.6)$ \\
Delay accident-ACLR & \\
mean \pm SD (range), month & \\
Preoperative side-to-side laxity (Rolimeter & \\
mean \pm SD (range), mm & $6.0 \pm 1.6(4-14)$ \\
Preoperative sport practice $n(\%)$ & \\
Pivoting & $105(34.2)$ \\
Pivoting-contact & $134(43.7)$ \\
Non-pivoting & $68(22.1)$ \\
Preoperative Tegner activity scale score & $6.0 \pm 1.2(3-9)$ \\
mean \pm SD (range) & \\
Follow-up & $35.2 \pm 11.0(14.3-50.2)$ \\
mean \pm SD (range), month & \\
\hline
\end{tabular}

${ }^{a} B M I$ body mass index, $A C L R$ anterior cruciate ligament reconstruction. $S A M B B A$ single-anteromedial bundle biological augmentation

The most frequent specific femoral button complication is soft tissue interposition, which is reported in 15 to $24.2 \%$ of patients [9, 15-17]. Mae et al. demonstrated a direct correlation with implant migration over time, however, this had no adverse clinical repercussions [15, 33]. Nevertheless, Gürpinar et al. pointed out that an interposition of soft tissues exceeding $3 \mathrm{~mm}$ increased the risk of implant migration and significantly decreased postoperative clinical scores [18]. These findings are, however, open to discussion, as they were based on the results of eight patients out of a cohort of 156. Positioning of the button posterior and distal to the epicondyle has also been identified as a risk factor for migration [33]. Given their frequency and potential clinical impact, any means of preventing these complications is welcome.

Intraoperative fluoroscopic control of the femoral button is the most frequently reported solution in the literature [9, $34,35]$. In the event of soft tissue interposition or improper deployment, a new attempt to correctly flip the implant can be attempted after the interposed tissue is bypassed [9]. A larger lateral surgical approach could also be performed over the guide pin to obtain direct visual control of the button [34]. Some authors have used this approach as an accessory arthroscopic portal, allowing its size to be limited while keeping the implant deployment visualized [19, 35]. Mistovitch et al. suggested using this portal routinely without
Table 2 Surgical characteristics

SAMBBA $n=307$

\begin{tabular}{|c|c|}
\hline \multicolumn{2}{|l|}{ Indication $n(\%)$} \\
\hline Primary ACLR ${ }^{\mathrm{a}}$ & $302(98.4)$ \\
\hline Revision ACLR $^{\mathrm{a}}$ & $5(1.6)$ \\
\hline \multicolumn{2}{|l|}{$\operatorname{LEAP}^{\mathrm{a}} n(\%)$} \\
\hline Modified lemaire & $14(4.6)$ \\
\hline ALLR $^{\mathrm{a}}$ & $12(3.9)$ \\
\hline \multicolumn{2}{|l|}{ Associated high tibial osteotomy $n(\%)$} \\
\hline Medial opening & $1(0.3)$ \\
\hline Anterior closing & $1(0.3)$ \\
\hline $\begin{array}{l}\text { Native ACL remnant preservation } \\
\text { mean } \pm \mathrm{SD} \text { (range), } \%\end{array}$ & $48.3 \pm 27.4(0-90)$ \\
\hline \multicolumn{2}{|l|}{ Meniscal tears $n(\%)$} \\
\hline Total & $143(46.5)$ \\
\hline Medial meniscus (MM) & $79(25.7)$ \\
\hline Lateral meniscus (LM) & $40(13.0)$ \\
\hline $\mathrm{MM}+\mathrm{LM}$ & $24(7.8)$ \\
\hline \multicolumn{2}{|c|}{ Cartilaginous lesions, ICRS ${ }^{\mathrm{a}}$ grading, $n(\%)$} \\
\hline \multicolumn{2}{|l|}{$\mathrm{MC}^{\mathrm{a}}$} \\
\hline $1-2$ & $13(4.2)$ \\
\hline $3-4$ & $7(2.3)$ \\
\hline \multicolumn{2}{|l|}{$\mathrm{LC}^{\mathrm{a}}$} \\
\hline $1-2$ & $2(0.6)$ \\
\hline $3-4$ & $2(0.6)$ \\
\hline \multicolumn{2}{|l|}{$\mathrm{FPC}^{\mathrm{a}}$} \\
\hline $1-2$ & $17(5.5)$ \\
\hline $3-4$ & $4(1.3)$ \\
\hline $\begin{array}{l}\text { Tourniquet time } \\
\text { mean } \pm \mathrm{SD} \text { (range), min }\end{array}$ & $30.2 \pm 8.2(0-81)$ \\
\hline
\end{tabular}

mean $\pm \mathrm{SD}$ (range), min

${ }^{\mathrm{a}} A C L R$ anterior cruciate ligament reconstruction, LEAP lateral extraarticular procedure, allr antero-lateral ligament reconstruction, ICRS International Cartilage Repair Society, $M C$ medial compartment, $L C$ lateral compartment, FPC femoro-patellar compartment, SAMBBA single-anteromedial bundle biological augmentation

prior fluoroscopic control [19]. According to Kang and Lee, using an outside-in femoral guide would allow the button to be easily found through this portal, in contrast with the transtibial or inside-out procedure [36].

These techniques are efficient and can limit potential complications related to the femoral button $[9,19,34$, 35]. However, intraoperative fluoroscopy is irradiating for both the patient and the surgeon and requires the involvement of trained personnel [37, 38]. Moreover, the use of an additional surgical approach is invasive and could increase the risk of sepsis. Both increase the overall surgical time. Checking the correct deployment of the implant by manual testing alone would prevent these pitfalls, but O'Brien et al. demonstrated the poor reliability of manual testing, with only $74.5 \%$ of femoral buttons having correct positioning [9]. Another option would be to check for proper passage of 
Fig. 3 Frontal, lateral and Schuss view radiographs following ACLR according to the SAMBBA technique. Female, 47-years old. Frontal, lateral and Schuss view radiographs at the nine-month follow-up (right knee). Correct deployment of the femoral button on the lateral cortex was verified on all radiographs. No soft tissue interposition was noticed

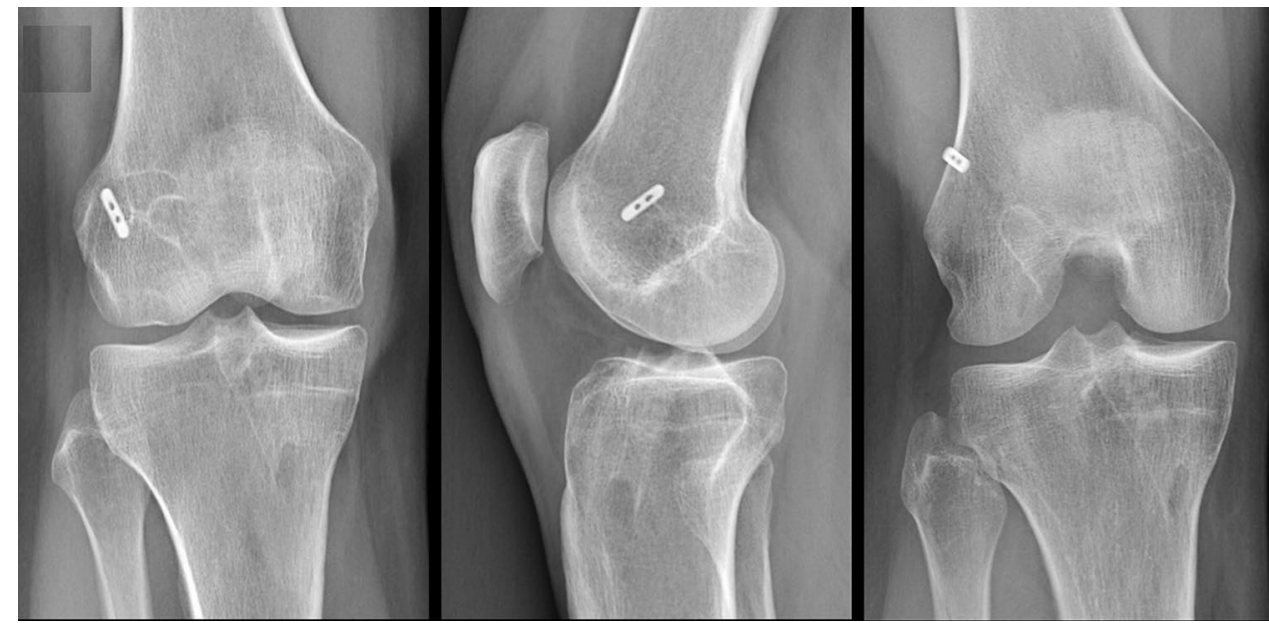

Table 3 Potentially femoral button-related complications

\begin{tabular}{ll}
\hline & SAMBBA $n=307$ \\
\hline Femoral button-related lateral pain $n(\%)$ & $9(2.9)$ \\
Lateral corticosteroid infiltration $n(\%)$ & $4(1.3)$ \\
$\begin{array}{l}\text { Delay ACLR - infiltration } \\
\text { mean } \pm \text { SD (range), month }\end{array}$ & $14.52 \pm 4.80(11.73-21.70)$ \\
Femoral button removal $n(\%)$ & $1(0.3)$ \\
Septic arthritis $n(\%)$ & 0 \\
\hline
\end{tabular}

SAMBBA single-anteromedial bundle biological augmentation

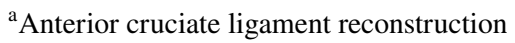

Table 4 Reoperations performed after ACLR (all reoperations were performed arthroscopically)

\begin{tabular}{ll}
\hline & SAMBBA $n=307$ \\
\hline Secondary arthroscopy: total $n(\%)$ & $15(4.9)$ \\
Meniscectomy $n(\%)$ & \\
$\quad$ Medial meniscus & $4(1.3)$ \\
$\quad$ Lateral meniscus & $1(0.3)$ \\
Surgery for cyclops syndrome & $8(2.6)$ \\
$n(\%)$ & \\
Revision ACLR & \\
$n(\%)$ & $2(0.7)$ \\
\hline
\end{tabular}

SAMBBA single-anteromedial bundle biological augmentation

${ }^{a}$ Anterior cruciate ligament reconstruction

the button through the femoral tunnel under intra-articular arthroscopic control. Nag and Gupta proposed pushing the implant through the tunnel using a guide pin under visual control until a loss of resistance occurred, indicating the passage of the femoral cortex [39]. However, this technique does not allow direct visualization of button deployment.

The present technique allows for this through direct arthroscopic control in the lateral gutter. It also prevents soft tissue interposition by systematic shaving of tissues along the lateral end of the femoral tunnel. Easy to handle and reproducible, it is both a nonirradiating and noninvasive solution. The main concern, although rare (nine patients, $2.9 \%$ ), was ITB irritation related to the implant. Most symptomatic patients (eight out of nine, $88.9 \%$ ) were successfully treated with medical treatment consisting of well-managed rehabilitation and ultrasound-guided corticosteroid infiltration in patients where symptoms persisted. Only one patient $(0.3 \%)$ required surgical removal of the implant, providing immediate relief of pain.

The retrospective design is the main limitation of the present study. However, a large sample size was obtained, and this is, to our knowledge, the largest cohort on the subject in the literature. Second, all data were collected from the SANTI study group database. Complications managed in other institutions were therefore not taken into account. However, all patients involved in the present study were followed up for at least 14.27 months after ACLR. The most feared complications, namely, septic arthritis and ITB irritation, would have occurred before this time. This also explains why a minimum of one year of follow-up seemed sufficient to us. Finally, the present study did not include a control group. The objective of this study was to demonstrate the reliability of intra-articular positioning of the femoral button. Therefore, comparison with another technique did not seem relevant, as only complications of this specific device were being examined.

\section{Conclusions}

Arthroscopic confirmation of femoral button deployment prevents soft tissue interposition without specific complications. This technique is reproducible, non-irradiating and non-invasive. 
Funding This article has no funding.

\section{Declarations}

Conflict of interest BSC and MT are Arthrex consultants. SG, AF and $\mathrm{AC}$ received a fellowship grant during the time this article was written.

Ethical approval Institutional Review Board Approval: COS-RGDS2021-01-004-SONNERYCOTTET-B.

\section{References}

1. Järvinen TLN, Alami GB, Karlsson J (2010) Anterior cruciate ligament graft fixation - a myth busted? Arthrosc J Arthrosc Relat Surg 26:681-684

2. Lubowitz JH, Guttmann D, Järvinen TLN, Kousa P, Kannus P, Järvinen M, Noyes FR, Stannard JP (2003) Letters to the Editor. Am J Sports Med 31:811-814

3. Kurosaka M, Yoshiya S, Andrish JT (1987) A biomechanical comparison of different surgical techniques of graft fixation in anterior cruciate ligament reconstruction. Am J Sports Med $15: 225-229$

4. Eysturoy NH, Nissen KA, Nielsen T, Lind M (2018) the influence of graft fixation methods on revision rates after primary anterior cruciate ligament reconstruction. Am J Sports Med 46:524-530

5. Lubowitz JH, Ahmad CS, Amhad CH, Anderson K (2011) Allinside anterior cruciate ligament graft-link technique: secondgeneration, no-incision anterior cruciate ligament reconstruction. Arthroscopy 27:717-727

6. Persson A, Kjellsen AB, Fjeldsgaard K, Engebretsen L, Espehaug B, Fevang JM (2015) Registry data highlight increased revision rates for endobutton/biosure HA in ACL reconstruction with hamstring tendon autograft: a nationwide cohort study from the Norwegian Knee Ligament Registry, 2004-2013. Am J Sports Med 43:2182-2188

7. Schurz M, Tiefenboeck TM, Winnisch M, Syre S, Plachel F, Steiner G, Hajdu S, Hofbauer M (2016) Clinical and functional outcome of all-inside anterior cruciate ligament reconstruction at a minimum of 2 years' follow-up. Arthroscopy 32:332-337

8. Karaoglu S, Halici M, Baktir A (2002) An unidentified pitfall of Endobutton use: case report. Knee Surg Sports Traumatol Arthrosc 10:247-249

9. O’Brien DF, Fones L, Stoj V, Edgar C, Coyner K, Arciero RA (2021) Confirming proper button deployment of suspensory fixation during ACL reconstruction. Orthop J Sports Med 9:2325967120974349

10. Baumfeld JA, Diduch DR, Rubino LJ, Hart JA, Miller MD, Barr MS, Hart JM (2008) Tunnel widening following anterior cruciate ligament reconstruction using hamstring autograft: a comparison between double cross-pin and suspensory graft fixation. Knee Surg Sports Traumatol Arthrosc 16:1108-1113

11. Fauno P, Kaalund S (2005) Tunnel widening after hamstring anterior cruciate ligament reconstruction is influenced by the type of graft fixation used: a prospective randomized study. Arthroscopy 21:1337-1341

12. Sabat D, Kundu K, Arora S, Kumar V (2011) Tunnel widening after anterior cruciate ligament reconstruction: a prospective randomized computed tomography-based study comparing 2 different femoral fixation methods for hamstring graft. Arthroscopy 27:776-783

13. Kawaguchi K, Kuribayashi S, Nakayama S, Nakazato K, Fukubayashi T, Okinaga S (2016) Lateral knee pain after outside-in anatomic double-bundle anterior cruciate ligament reconstruction using the tightrope RT. Knee Surg Relat Res 28:83-87

14. Taketomi S, Inui H, Hirota J, Nakamura K, Sanada T, Masuda H, Tanaka S, Nakagawa T (2013) Iliotibial band irritation caused by the EndoButton after anatomic double-bundle anterior cruciate ligament reconstruction: report of two cases. Knee 20:291-294

15. Mae T, Kuroda S, Matsumoto N, Yoneda M, Nakata K, Yoshikawa H, Shino K (2011) Migration of EndoButton after anatomic double-bundle anterior cruciate ligament reconstruction. Arthroscopy 27:1528-1535

16. Simonian PT, Behr CT, Stechschulte DJ, Wickiewicz TL, Warren RF (1998) Potential pitfall of the EndoButton. Arthroscopy 14:66-69

17. Taketomi S, Inui H, Nakamura K, Yamagami R, Tahara K, Sanada T, Masuda H, Tanaka S, Nakagawa T (2015) Secure fixation of femoral bone plug with a suspensory button in anatomical anterior cruciate ligament reconstruction with bone-patellar tendon-bone graft. Joints 3:102-108

18. Gürpınar T, Polat B, Eren M, Çarkçı E, Özyalvaç ON, Erdoğan S (2020) The effect of soft tissue interposition of the Endobutton on clinical results and on its postoperative migration after single-bundle anterior cruciate ligament reconstruction. Knee 27:1980-1987

19. Mistovich RJ, O’Toole POJ, Ganley TJ (2014) Pediatric anterior cruciate ligament femoral fixation: the trans-iliotibial band endoscopic portal for direct visualization of ideal button placement. Arthrosc Tech 3:e335-338

20. Sonnery-Cottet B, Freychet B, Murphy CG, Pupim BHB, Thaunat M (2014) Anterior cruciate ligament reconstruction and preservation: the single-anteromedial bundle biological augmentation (SAMBBA) technique. Arthrosc Tech 3:e689-693

21. Sonnery-Cottet B, Rezende FC, Martins Neto A, Fayard JM, Thaunat M, Kader DF (2014) Arthroscopically confirmed femoral button deployment. Arthrosc Tech 3:e309-312

22. Papachristou G, Nikolaou V, Efstathopoulos N, Sourlas J, Lazarettos J, Frangia K, Papalois A (2007) ACL reconstruction with semitendinosus tendon autograft without detachment of its tibial insertion: a histologic study in a rabbit model. Knee Surg Sports Traumatol Arthrosc 15:1175-1180

23. Kim S-G, Kurosawa H, Sakuraba K, Ikeda H, Takazawa S, Takazawa Y (2005) Development and application of an insideto-out drill bit for anterior cruciate ligament reconstruction. Arthroscopy 21:1012

24. Jesani S, Getgood A (2019) Modified lemaire lateral extraarticular tenodesis augmentation of anterior cruciate ligament reconstruction. JBJS Essent Surg Tech 9(4):e41.1

25. Kim HS, Seon JK, Jo AR (2013) Current trends in anterior cruciate ligament reconstruction. Knee Surg Relat Res 25:165-173

26. Kong C-G, In Y, Kim G-H, Ahn C-Y (2012) Cross pins versus endobutton femoral fixation in hamstring anterior cruciate ligament reconstruction: minimum 4-year follow-up. Knee Surg Relat Res 24:34-39

27. Saccomanno MF, Shin JJ, Mascarenhas R, Haro M, Verma NN, Cole BJ, Bach BR (2014) Clinical and functional outcomes after anterior cruciate ligament reconstruction using cortical button fixation versus transfemoral suspensory fixation: a systematic review of randomized controlled trials. Arthroscopy 30:1491-1498

28. Choi N-H, Oh J-S, Jung S-H, Victoroff BN (2013) Correlation between endobutton loop length and tunnel widening after hamstring anterior cruciate ligament reconstruction. Am J Sports Med 41:101-106

29. Ma CB, Francis K, Towers J, Irrgang J, Fu FH, Harner CH (2004) Hamstring anterior cruciate ligament reconstruction: a comparison of bioabsorbable interference screw and endobutton-post fixation. Arthroscopy 20:122-128 
30. Pioger C, Saithna A, Rayes J, Haidar IM, Fradin T, Ngbilo C, Vieira TD, Cavaignac E, Sonnery-Cottet B (2021) Influence of preoperative tunnel widening on the outcomes of a single stageonly approach to every revision anterior cruciate ligament reconstruction: an analysis of 409 consecutive patients from the SANTI study group. Am J Sports Med 49(6):1431-1440

31. Muneta T, Yagishita K, Kurihara Y, Sekiya I (1999) Intra-articular detachment of the Endobutton more than 18 months after anterior cruciate ligament reconstruction. Arthroscopy 15:775-778

32. Yanmiș I, Tunay S, Oğuz E, Yildiz C, Ozkan H, Kirdemir V (2004) Dropping of an EndoButton into the knee joint 2 years after anterior cruciate ligament repair using proximal fixation methods. Arthroscopy 20:641-643

33. Uchida R, Mae T, Matsumoto N, Kuroda S, Toritsuka Y, Shino $\mathrm{K}$ (2014) The effect of cortical button location on its post-operative migration in anatomical double-bundle anterior cruciate ligament reconstruction. Knee Surg Sports Traumatol Arthrosc 22:1047-1054

34. Balldin BC, Nuelle CW, DeBerardino TM (2020) Is intraoperative fluoroscopy necessary to confirm device position for femoralsided cortical suspensory fixation during anterior cruciate ligament reconstruction? J Knee Surg 33:265-269

35. Ohnishi Y, Chang A, Utsunomiya H, Suzuki H, Nakamura E, Sakai A, Uchida S (2017) Arthroscopic technique to reduce suture button migration during anterior cruciate ligament reconstruction procedure. Arthrosc Tech 6:e1927-e1931
36. Kang SG, Lee YS (2017) Arthroscopic control for safe and secure seating of suspensory devices for femoral fixation in anterior cruciate ligament reconstruction using three different techniques. Knee Surg Relat Res 29:33-38

37. Hayda RA, Hsu RY, DePasse JM, Gil JA (2018) Radiation exposure and health risks for orthopaedic surgeons. J Am Acad Orthop Surg 26:268-277

38. Singer G (2005) Occupational radiation exposure to the surgeon. J Am Acad Orthop Surg 13:69-76

39. Nag HL, Gupta H (2012) Seating of tightrope RT button under direct arthroscopic visualization in anterior cruciate ligament reconstruction to prevent potential complications. Arthrosc Tech $1: \mathrm{e} 83-85$

40. von Elm E, Altman DG, Egger M, Pocock SJ, Gøtzsche PC, Vandenbroucke JP, Initiative STROBE (2007) The strengthening the reporting of observational studies in epidemiology (STROBE) statement: guidelines for reporting observational studies. Lancet 370:1453-1457

Publisher's Note Springer Nature remains neutral with regard to jurisdictional claims in published maps and institutional affiliations. 